\title{
TENSOR PRODUCTS OF MAXIMAL ABELIAN SUBALGBERAS OF C*-ALGEBRAS
}

\author{
SIMON WASSERMANN \\ Department of Mathematics, University of Glasgow, Glasgow G12 8QW, United Kingdom \\ e-mail:asw@maths.gla.ac.uk
}

(Received 10 May, 2007; revised 27 August, 2007 and 23 November, 2007; accepted 30 November, 2007)

\begin{abstract}
It is shown that if $C_{1}$ and $C_{2}$ are maximal abelian self-adjoint subalgebras (masas) of $\mathrm{C}^{*}$-algebras $A_{1}$ and $A_{2}$, respectively, then the completion $C_{1} \otimes C_{2}$ of the algebraic tensor product $C_{1} \odot C_{2}$ of $C_{1}$ and $C_{2}$ in any $\mathrm{C}^{*}$-tensor product $A_{1} \otimes_{\beta} A_{2}$ is maximal abelian provided that $C_{1}$ has the extension property of Kadison and Singer and $C_{2}$ contains an approximate identity for $A_{2}$. Examples are given to show that this result can fail if the conditions on the two masas do not both hold. This gives an answer to a long-standing question, but leaves open some other interesting problems, one of which turns out to have a potentially intriguing implication for the Kadison-Singer extension problem.
\end{abstract}

2000 Mathematics Subject Classification. 46L06.

1. Introduction. If $A_{1}$ and $A_{2}$ are $C^{*}$-algebras with centres $Z_{1}$ and $Z_{2}$, respectively, it was shown by Richard Haydon and the author [6] that the centre of the minimal $\mathrm{C}^{*}$ tensor product $A_{1} \otimes_{\min } A_{2}$ is just the closure of the algebraic tensor product $Z_{1} \odot Z_{2}$ in $A_{1} \otimes_{\min } A_{2}$. This closure is naturally isomorphic to $Z_{1} \otimes_{\min } Z_{2}$. The result was also shown to follow from a more general slice map result [12, Theorem 4] and the analogous result for arbitrary $C^{*}$-tensor products was subsequently established by Archbold using the Dixmier approximation property [3]. Batty later gave a neat alternative proof of Archbold's general result [5].

Analogous questions arise for maximal abelian self-adjoint $\mathrm{C}^{*}$-subalgebras (or masas) of $\mathrm{C}^{*}$-algebras. If $C_{1}$ and $C_{2}$ are masas of $A_{1}$ and $A_{2}$, respectively, then the closure of the algebraic tensor product $C_{1} \odot C_{2}$ in any $\mathrm{C}^{*}$-completion $A_{1} \otimes_{\beta} A_{2}$ of $A_{1} \odot A_{2}$ is naturally isomorphic to $C_{1} \otimes_{\min } C_{2}$ since the algebras in the tensor product are abelian. The slice map result [12, Theorem 4] implies that $C_{1} \otimes_{\min } C_{2}$ is again a masa in $A_{1} \otimes_{\min } A_{2}$, and it is natural to ask whether $C_{1} \otimes_{\min } C_{2}$ is a masa in $A_{1} \otimes_{\beta} A_{2}$ for any $\mathrm{C}^{*}$-norm $\beta$ on $A_{1} \odot A_{2}$ other than the minimal norm when $A_{1} \odot A_{2}$ has more than one $\mathrm{C}^{*}$-norm.

Although this question was originally raised in [12], up to now little progress seems to have been made. In this paper we give a solution to the problem. There are two main results. The first, a positive one, states that if one of the masas $C_{1}, C_{2}$ possesses the Kadison-Singer extension property and the other contains an approximate identity for the ambient algebra, then the question has a positive answer. The second, a negative answer to the general question, is a pair of example of masas $C_{1}$ and $C_{2}$ of $\mathrm{C}^{*}$-algebras $A_{1}$ and $A_{2}$, respectively, such that $C_{1} \otimes_{\min } C_{2}$ is not maximal abelian in $A_{1} \otimes_{\max } A_{2}$ in each case. Connections with the Kadison-Singer extension problem [9] of whether 
$\ell^{\infty}(\mathbb{N})$ has the extension property relative to $B\left(\ell^{2}(\mathbb{N})\right.$ will be discussed in the final section.

2. Masas with the extension property. A masa $C$ of a $C^{*}$-algebra $A$ is said to have the extension property (see [9], [2], [4]) if

(i) any pure state (i.e. character) of $C$ has a unique pure state extension to $A$ and (when $A$ is non-unital).

(ii) no pure state of $A$ annihilates $C$.

Condition (ii) is well-known to be equivalent to the condition (see [1, Proof of Lemma 2.32])

(ii)' $C$ contains an increasing approximate identity for $A$.

It is a straightforward consequence of the Krein-Milman theorem that any pure state of a masa $C$ with the extension property has a unique state extension to $A$. An alternative characterisation [4] of the extension property for unital $A$ states that, if $\mathcal{U}(C)$ denotes the unitary group of $C$, then for any $x \in A$ the intersection $C \cap \overline{\operatorname{co}}\left\{u x u^{*}: u \in \mathcal{U}(C)\right\}$ of $C$ with the closed convex hull $\overline{\mathrm{co}}\left\{u x u^{*}: u \in \mathcal{U}(C)\right\}$ contains exactly one point.

EXAMPLES 1. In the reduced $C^{*}$-algebra $C_{r}^{*}\left(\mathbb{F}_{2}\right)$ of the free group on two generators, with $u$ and $v$ the canonical unitary generators, the abelian $\mathrm{C}^{*}$-subalgebras generated by $u$ and $v$, respectively, are masas with the extension property [4, Example (i)]. Moreover $C_{r}^{*}\left(\mathbb{F}_{2}\right)$ is not nuclear [11]; (see also [14]).

2. No non-atomic masa of $B\left(\ell^{2}(\mathbb{N})\right)$ has the extension property [9].

In what follows the minimal $C^{*}$-tensor product of two $C^{*}$-algebras will be denoted by $A \otimes B$ when at least one of $A$ and $B$ is abelian. The following well-known factorisation result for states on a tensor product will be required in the proof of Theorem 2.

Lemma 1 [5, Lemma 3]. Let $\varphi$ be a state on $A_{1} \otimes_{\beta} A_{2}$ such that the restriction $\varphi_{1}$ of $\varphi$ to $A_{1}$ is a pure state of $A_{1}$. Then $\varphi=\varphi_{1} \otimes \varphi_{2}$ for some state $\varphi_{2}$ on $A_{2}$.

In the proof of the following result, which is analogous to that of [5, Theorem 4], the unitization $\tilde{A}$ of $A$ will be taken to be the subalgebra $A+\mathbb{C} .1$ of the multiplier algebra $M(A)$, so that $\tilde{A}=A$ if $A$ is itself unital. If $\beta$ is a $\mathrm{C}^{*}$-norm on $A_{1} \odot A_{2}, \tilde{\beta}$ will denote the unique $\mathrm{C}^{*}$-norm on $\tilde{A_{1}} \odot \tilde{A_{2}}$ extending $\beta$; (see $[8]$ ).

Theorem 2. Let $A_{1}$ and $A_{2}$ be $C^{*}$-algebras with $A_{2}$ unital and let $C$ be a masa of $A_{1}$ with the extension property. Then in any $C^{*}$-completion $A_{1} \otimes_{\beta} A_{2}$,

$$
(C \otimes 1)^{c}=C \otimes A_{2},
$$

where $(C \otimes 1)^{c}=\left\{x \in A_{1} \otimes_{\beta} A_{2}: x(c \otimes 1)=(c \otimes 1) x\right.$ for all $\left.c \in C\right\}$.

Proof. 1. Assume first that $A_{1}$ is unital. If $\Phi: A_{1} \otimes_{\beta} A_{2} \rightarrow A_{1} \otimes_{\min } A_{2}$ is the canonical homomorphism, its restriction to $C \otimes A_{2}$ is an isomorphism and $C \otimes A_{2}$ can be identified with its image in $A_{1} \otimes_{\min } A_{2}$ under $\Phi$. With this identification $\left.\Phi\right|_{C_{1} \otimes A_{2}}$ is just the identity map. If $x \in(C \otimes 1)^{c}$, then $[x, C \otimes 1]=0$, which implies that $[\Phi(x), C \otimes$ 1] $=0$. By [12, Theorem 4], $\Phi(x) \in C \otimes A_{2}$. Replacing $x$ by $(x-\Phi(x))^{*}(x-\Phi(x))$, it is sufficient to show that if $x \geq 0$ and $\Phi(x)=0$ then $x=0$. 
With these assumptions $\|x\| \in \mathrm{Sp}(x),\|x\| 1-x$ is singular and the closed left ideal $I$ of $D=(C \otimes 1)^{c}$ generated by $\|x\| 1-x$ is proper. Let

$$
J=\{c \in C: c \otimes 1 \in I\} .
$$

Since $I(C \otimes 1) \subseteq I, J$ is a proper closed two-sided ideal of $C$ and since $J \otimes 1=(C \otimes$ 1) $\cap I$, there are canonical isometric isomorphisms

$$
C / J \cong(C \otimes 1) /(J \otimes 1) \cong(C \otimes 1+I) / I,
$$

by $[\mathbf{1 0}, 1.17 .6]$. Let $\chi$ be a character of $C$ that annihilates $J$. Via these isomorphisms, $\chi$ corresponds to a linear functional $\varphi$ on $(C \otimes 1+I) / I$ such that $\|\varphi\|=1$ and $\varphi(1+$ $I)=1$. By the Hahn-Banach theorem $\varphi$ extends to a linear functional of norm 1 on $D / I$ which, when composed with the quotient map, gives a state $\bar{\varphi}$ on $D$ such that $\bar{\varphi}(c \otimes 1)=\chi(c)$ for $c \in C$. Let $\psi$ be an extension of $\bar{\varphi}$ to a state on $A_{1} \otimes_{\beta} A_{2}$. Letting $\psi_{1}$ be the restriction of $\psi$ to $A_{1}$, so that $\psi_{1}(a)=\psi(a \otimes 1), \psi_{1}$ is a pure state since $C$ has the extension property. By Lemma $1, \psi=\psi_{1} \otimes \psi_{2}$ for some state $\psi_{2}$ on $A_{2}$. Now $\psi(\|x\| 1-x)=0$, since $\left.\psi\right|_{I}=0$, and $\psi(x)=\left(\psi_{1} \otimes \psi_{2}\right)(\Phi(x))=0$. Thus $\|x\|=\psi(\|x\| 1-x)=0$, which implies that $x=0$, as required.

2. If $A_{1}$ is not unital, let $\tilde{A_{1}}$ be the unitisation of $A_{1}$ and let $\tilde{C}=C+\mathbb{C} 1$. Then $\tilde{C}$ is a masa in $\tilde{A_{1}}$. To see that $\tilde{C}$ has the extension property in $\tilde{A_{1}}$, let $f$ be a pure state of $\tilde{C}$ and let $\bar{f}$ be a pure state extension of $f$ to $\tilde{A_{1}}$. If $f$ is the unique pure state annihilating $C$, then $g=\left.\bar{f}\right|_{A_{1}}=0$, since otherwise $g$ would be a pure state of $A_{1}$ that annihilated $C$. In this case $\bar{f}$ is the unique pure state of $\tilde{A}_{1}$ that annihilates $A_{1}$. If $\left.f\right|_{C} \neq 0$, then $\left.f\right|_{C}$ is a pure state of $C$ and $\left.\bar{f}\right|_{A_{1}}$ is a pure state extension of $\left.f\right|_{C}$ and so uniquely determined by $f$. Since $\bar{f}$ is uniquely determined by its restriction to $A_{1}$, it follows that $\bar{f}$ is uniquely determined by $f$.

For $x \in A_{1} \otimes_{\beta} A_{2}$, if $x \in(C \otimes 1)^{c}$ then $x \in(\tilde{C} \otimes 1)^{c} \subseteq \tilde{A}_{1} \otimes_{\tilde{\beta}} A_{2}$, which implies by part 1 that $x \in \tilde{C} \otimes A_{2}$. Let $\varphi$ be the state on $\tilde{A_{1}}$ that annihilates $A_{1}$. Then $\chi=\left.\varphi\right|_{\tilde{C}}$ is the character of $\tilde{C}$ that annihilates $C$ and the kernel of the map $\chi \otimes i d_{A_{2}}$ is just $C \otimes A_{2}$. Since $0=\left(\varphi \otimes i d_{A_{2}}\right)(x)=\left(\chi \otimes i d_{A_{2}}\right)(x)$, it follows that $x \in C \otimes A_{2}$, as required.

Corollary 3. Let $A_{1}$ and $A_{2}$ be $C^{*}$-algebras and let $C_{1}$ and $C_{2}$ be masas of $A_{1}$ and $A_{2}$, respectively, such that $C_{1}$ has the extension property and $C_{2}$ contains an approximate identity for $A_{2}$ if $A_{2}$ is not unital. Then $C_{1} \otimes C_{2}$ is a masa of $A_{1} \otimes_{\beta} A_{2}$ for any $C^{*}$-norm $\beta$ on $A_{1} \odot A_{2}$. Moreover $C_{1} \otimes C_{2}$ has the extension property if and only if $C_{2}$ does.

Proof. Assume that $C_{1}$ has the extension property. If $A_{2}$ is unital, it is immediate from Theorem 2 that $\left(C_{1} \otimes C_{2}\right)^{c} \subseteq\left(C_{1} \otimes 1\right)^{c}=C_{1} \otimes A_{2}$. If $A_{2}$ is non-unital and $\left\{e_{\lambda}\right\}$ is an approximate identity of $A_{2}$ in $C_{2}$, for $x \in\left(C_{1} \otimes C_{2}\right)^{c},\left[x, c \otimes e_{\lambda}\right]=0$ for any $c \in C_{1}$ and any $\lambda$. Since $x(c \otimes 1)=\lim _{\lambda} x\left(c \otimes e_{\lambda}\right)$ and $(c \otimes 1) x=\lim _{\lambda}\left(c \otimes e_{\lambda}\right) x$, it follows that, as an element of $A_{1} \otimes_{\tilde{\beta}} \tilde{A_{2}}, x$ lies in $\left(C_{1} \otimes 1\right)^{c}$, which equals $C_{1} \otimes_{\tilde{\beta}} \tilde{A_{2}}$, the closure of $C_{1} \odot \tilde{A_{2}}$ in $A_{1} \otimes_{\tilde{\beta}} \tilde{A_{2}}$, by Theorem 2. Since $C_{1}$ is abelian, $C_{1} \otimes_{\tilde{\beta}} \tilde{A_{2}}$ identifies naturally with $C_{1} \otimes \tilde{A_{2}}$ and so $x \in\left(C_{1} \otimes \tilde{A_{2}}\right) \cap\left(A_{1} \otimes_{\beta} A_{2}\right)$. Let $\Psi$ denote the canonical homomorphism from $A_{1} \otimes_{\tilde{\beta}} \tilde{A_{2}}$ to $A_{1} \otimes_{\min } \tilde{A_{2}}$. Then $\left.\Psi\right|_{C_{1} \otimes \tilde{A}_{2}}$ is the identity map on $C_{1} \otimes \tilde{A_{2}}$, since $C_{1} \otimes_{\tilde{\beta}} \tilde{A}_{2}$ and $C_{1} \otimes \tilde{A_{2}}$ are naturally identified, and $\Psi(x)$ is in $\left(C_{1} \otimes \tilde{A}_{2}\right) \cap\left(A_{1} \otimes_{\min } A_{2}\right)$, which equals $C_{1} \otimes A_{2}$ by [12, Corollary 5] (or a simple slice map argument), since $C_{1}$ is abelian. Thus $x \in C_{1} \otimes_{\beta} A_{2}$. If $X$ is the spectrum of $C_{1}$, there is a natural isomorphism $C_{1} \otimes A_{2} \cong C_{0}\left(X, A_{2}\right)$. Via this isomorphism $C_{1} \otimes C_{2}$ identifies with $C_{0}\left(X, C_{2}\right)$ and $x$ identifies with a function $\bar{x}$ in $C_{0}\left(X, A_{2}\right)$. For $\chi \in X$ 
and $c \in C_{2}$, there is a function $f \in C_{0}\left(X, C_{2}\right)$ such that $f(\chi)=c$. Since $x \in\left(C_{1} \otimes C_{2}\right)^{c}$, $[\bar{x}(\chi), c]=[\bar{x}, f](\chi)=0$, which implies that $\bar{x}(\chi) \in C_{2}$ for $\chi \in X$ and so $\bar{x} \in C_{0}\left(X, C_{2}\right)$. Thus $x \in C_{1} \otimes C_{2}$, as required.

Now assume that $C_{1}$ and $C_{2}$ have the extension property and let $\varphi$ be a character of $C_{1} \otimes C_{2}$. Then $\varphi=\varphi_{1} \otimes \varphi_{2}$ for suitable characters $\varphi_{1}$ and $\varphi_{2}$ of $C_{1}$ and $C_{2}$, respectively. Let $\psi_{i}$ be the unique pure state extension of $\varphi_{i}$ to $A_{i}$ for $i=1,2$ and let $\psi$ be the pure state $\psi_{1} \otimes \psi_{2}$ of $A_{1} \otimes_{\beta} A_{2}$. If $\bar{\varphi}$ is any pure state extension of $\varphi$ to $A \otimes_{\beta} A_{2}$, and $\bar{\varphi}_{1}$ and $\bar{\varphi}_{2}$ are the restrictions of $\bar{\varphi}$ to $A_{1}$ and $A_{2}$, respectively, then $\left.\bar{\varphi}_{i}\right|_{C_{i}}=\varphi_{i}$, which implies that $\bar{\varphi}_{i}=\psi_{i}$ for $i=1,2$. By Lemma $1, \bar{\varphi}=\psi_{1} \otimes \psi_{2}=\psi$ and condition (i) in the definition of the extension property holds. If $A_{1}$ or $A_{2}$ is non-unital, it is an immediate consequence of condition (ii)' in the definition of the extension property that condition (ii)' holds for $C_{1} \otimes C_{2}$ relative to $A_{1} \otimes_{\beta} A_{2}$. Thus $C_{1} \otimes C_{2}$ has the extension property. Conversely, if $C_{1} \otimes C_{2}$ has the extension property, it is a simple exercise using similar methods to show that $C_{2}$ has the extension property.

Note. 1. An alternative proof of Theorem 2 when $A_{1}$ is unital can be given using the characterisation of the extension property in terms of unitary conjugates given in the paragraph following condition (ii)' at the beginning of this section.

2. If both $C_{1}$ and $C_{2}$ have the extension property a more direct proof of the first part of Corollary 3 can be given as follows. If $C$ is an abelian C*-subalgebra of $A_{1} \otimes_{\beta} A_{2}$ containing $C_{1} \otimes C_{2}$, let $\varphi=\varphi_{1} \otimes \varphi_{2}$ be a character of $C_{1} \otimes C_{2}$. If $\bar{\varphi}$ is a character of $C$ extending $\varphi$, then $\bar{\varphi}$ extends to a pure state $\psi$ of $A_{1} \otimes_{\beta} A_{2}$ which equals $\psi_{1} \otimes \psi_{2}$, where $\psi_{i}$ is the unique state extension of $\varphi_{i}$ to $A_{i}$ for $i=1,2$, by the argument of the second paragraph of the above proof. Thus $\bar{\varphi}=\left.\left(\psi_{1} \otimes \psi_{2}\right)\right|_{C}$, which means that $\varphi$ has a unique character extension to $C$. Moreover no pure state of $A_{1} \otimes_{\beta} A_{2}$ and hence no character of $C$ has a restriction to $C_{1} \otimes C_{2}$ equal to 0 , since $C_{1} \otimes C_{2}$ contains an approximate identity for $A_{1} \otimes_{\beta} A_{2}$. It follows by the Stone-Weierstrass theorem that $C=$ $C_{1} \otimes C_{2}$.

\section{Masas with tensor products which are not maximal abelian.}

3.1. Let $A=C_{r}^{*}\left(\mathbb{F}_{2}\right)+K\left(\ell^{2}\left(\mathbb{F}_{2}\right)\right)$ in $B\left(\ell^{2}\left(\mathbb{F}_{2}\right)\right)$, where $K\left(\ell^{2}\left(\mathbb{F}_{2}\right)\right)$ denotes the compact linear operators on $\ell^{2}\left(\mathbb{F}_{2}\right)$. Then $A$ is a $\mathrm{C}^{*}$-algebra, $K\left(\ell^{2}\left(\mathbb{F}_{2}\right)\right)$ is an ideal of $A$ and $A / K\left(\ell^{2}\left(\mathbb{F}_{2}\right)\right) \cong C_{r}^{*}\left(\mathbb{F}_{2}\right)$. Let $q$ be the canonical quotient map from $A$ onto $C_{r}^{*}\left(\mathbb{F}_{2}\right)$, and let $\lambda$ and $\rho$ denote the representations of $C_{r}^{*}\left(\mathbb{F}_{2}\right)$ corresponding to the leftand right-regular representations of $\mathbb{F}_{2}$ on $\ell^{2}\left(\mathbb{F}_{2}\right)$, respectively. Then $\{\lambda \circ q, \rho \circ q\}$ is a commuting pair of representations of the pair $\{A, A\}$ on $\ell^{2}\left(\mathbb{F}_{2}\right)$ with corresponding representation $\pi_{r}$ of $A \odot A$ given by

$$
\pi_{r}\left(\sum a_{i} \otimes b_{i}\right)=\sum \lambda\left(q\left(a_{i}\right)\right) \rho\left(q\left(b_{i}\right)\right)
$$

A C*-norm \|\|$_{\alpha}$ on $A \odot A$ is defined by

$$
\|x\|_{\alpha}=\max \left\{\|x\|_{\min },\left\|\pi_{r}(x)\right\|\right\} \quad(x \in A \odot A) .
$$

Let $\left\{\xi_{g}: g \in \mathbb{F}_{2}\right\}$ be the canonical orthonormal basis of $\ell^{2}\left(\mathbb{F}_{2}\right)$, for each $g \in \mathbb{F}_{2}$ let $e_{g}$ be the projection onto the one dimensional subspace $\mathbb{C} \xi_{g}$ and let $C$ be the abelian $\mathrm{C}^{*}$-algebra generated by $\left\{e_{g}: g \in \mathbb{F}_{2}\right\} \cup\{1\}$. Then $C \subset K\left(\ell^{2}\left(\mathbb{F}_{2}\right)\right)+\mathbb{C} .1 \subset A$. 
Proposition 4. The algebra $C$ is maximal abelian in $A$, but $C \otimes C$ is not maximal abelian in $A \otimes_{\beta}$ A for any $C^{*}$-norm \|\|$_{\beta}$ satisfying $\|x\|_{\beta} \geq\|x\|_{\alpha}$ on $x \in A \odot A$, and in particular if \|\|$_{\beta}=\|\|_{\max }$.

Proof. 1. To see that $C$ is maximal abelian in $A$, let $L$ be the closure of $C$ in the weak operator topology. Then $L$ is a maximal abelian $*$-subalgebra of $B\left(\ell^{2}\left(\mathbb{F}_{2}\right)\right)$ isomorphic to $\ell^{\infty}(\mathbb{N})$ and the canonical projection $\sigma$ from $B\left(\ell^{2}\left(\mathbb{F}_{2}\right)\right)$ onto $L$ is given by

$$
\sigma(x)=\sum_{g \in \mathbb{F}_{2}} e_{g} x e_{g}
$$

for $x \in B\left(\ell^{2}\left(\mathbb{F}_{2}\right)\right)$, the convergence of the sum on the right being in the strong operator topology. If $x \in C^{c}=C^{\prime} \cap A$, then $\sigma(x)=\sum_{g \in \mathbb{F}_{2}} e_{g} x e_{g}=\sum_{g \in \mathbb{F}_{2}} x e_{g}=x$. Now $x=$ $k+a$, where $k \in K\left(\ell^{2}\left(\mathbb{F}_{2}\right)\right)$ and $a \in C_{r}^{*}\left(\mathbb{F}_{2}\right)$. Since $e_{g} \lambda_{h} e_{g}=0$ for $g, h \in \mathbb{F}_{2}$ when $h$ is not the identity of $\mathbb{F}_{2}, \sigma(a) \in \mathbb{C} .1$ and since $\sigma(k) \in C$, it follows that $\sigma(x) \in C$. Thus $C^{c}=C$.

2. By $[\mathbf{1 1}]$ (see also [13]) the representation of $C_{r}^{*}\left(\mathbb{F}_{2}\right) \odot C_{r}^{*}\left(\mathbb{F}_{2}\right)$ on $\ell^{2}\left(\mathbb{F}_{2}\right)$ given by

$$
\sum a_{i} \otimes b_{i} \rightarrow \sum \lambda\left(a_{i}\right) \rho\left(b_{i}\right)
$$

is not continuous relative to \|\|$_{\min }$, which implies that there is a non-zero element in the kernel of the canonical homomorphism from $A \otimes_{\alpha} A$ to $A \otimes_{\min } A$ and hence a non-zero element $x$ in the kernel of the canonical homomorphism $\Psi$ from $A \otimes_{\beta} A$ to $A \otimes_{\min } A$. Since $K\left(\ell^{2}\left(\mathbb{F}_{2}\right)\right)$ is nuclear, the restriction of \|\|$_{\beta}$ to $K\left(\ell^{2}\left(\mathbb{F}_{2}\right)\right) \odot A$ coincides with \|\|$_{\min }$ and $\left.\Psi\right|_{K\left(\ell^{2}\left(\mathbb{F}_{2}\right)\right) \otimes_{\beta} A}$ is isometric. Thus for $k \in K\left(\ell^{2}\left(\mathbb{F}_{2}\right)\right)$,

$$
\|x(k \otimes 1)\|_{\beta}=\|\Psi(x(k \otimes 1))\|_{\min }=\|\Psi(x) \Psi(k \otimes 1)\|_{\min }=0,
$$

which implies that $x(k \otimes 1)=0$. Similarly $(k \otimes 1) x=0$. For $c \in C$ with $c=k+\lambda 1$, where $k \in K\left(\ell^{2}\left(\mathbb{F}_{2}\right)\right)$ and $\lambda \in \mathbb{C}$,

$$
[x, c \otimes 1]=[x, k \otimes 1]=0,
$$

so that $x \in(C \otimes 1)^{c}$. Similarly $x \in(1 \otimes C)^{c}$, so that $x \in(C \otimes C)^{c}$. Since $\Psi(x)=0$ and $\left.\Psi\right|_{C \otimes C}$ is isometric, $x \notin C \otimes C$, which means that $C \otimes C$ is not maximal abelian in $A \otimes_{\beta} A$.

3.2. This result shows that without the requirement in the statement of Corollary 3 that $C_{1}$ have the extension property, $C_{1} \otimes C_{2}$ may not be maximal abelian in $A_{1} \otimes_{\beta} A_{2}$. By modifying the construction of the $\mathrm{C}^{*}$-algebra $A$ above, it also follows that the conclusion of the Corollary can fail if $C_{2}$ does not contain an approximate identity for $A_{2}$.

To see this, let $H$ be a separable infinite dimensional Hilbert space with orthonormal basis $\left\{\xi_{i}: i \in \mathbb{N}\right\}$, and let $H_{1}$ and $H_{2}$ be the closures of the linear subspaces of $H$ spanned by $\left\{\xi_{2 i}: i \in \mathbb{N}\right\}$ and $\left\{\xi_{2 i-1}: i \in \mathbb{N}\right\}$, respectively. Then $H=H_{1} \oplus H_{2}$. A self-adjoint unitary operator $u$ on $H$ is defined by

$$
u \xi_{2 i}=\frac{1}{\sqrt{2}}\left(\xi_{2 i}+\xi_{2 i-1}\right), \quad u \xi_{2 i-1}=\frac{1}{\sqrt{2}}\left(\xi_{2 i}-\xi_{2 i-1}\right)
$$


Let $\left\{e_{i j}\right\}$ be the set of rank one matrix units associated with the basis $\left\{\xi_{i}\right\}$. Then

$$
u=\sum_{i} \frac{1}{\sqrt{2}}\left(e_{2 i, 2 i}+e_{2 i, 2 i-1}+e_{2 i-1,2 i}-e_{2 i-1,2 i-1}\right),
$$

the sum on the right hand side converging in the strong operator topology. If $t \in B(H)$ is such that $t H_{1} \subset H_{1}$ and $\left.t\right|_{H_{2}}=0$, then

$$
t=\sum_{i, j} t_{i j} e_{2 i, 2 j}
$$

where $t_{i j}=\left(t \xi_{2 j} \mid \xi_{2 i}\right)$, the convergence again being in the strong operator topology. Then

$$
u t u=\frac{1}{2} \sum_{i, j} t_{i j}\left(e_{2 i, 2 j}+e_{2 i, 2 j-1}+e_{2 i-1,2 j}+e_{2 i-1,2 j-1}\right) .
$$

Let $B=C_{r}^{*}\left(\mathbb{F}_{2}\right) \oplus\{0\}$ on $H$, where $C_{r}^{*}\left(\mathbb{F}_{2}\right)$ is acting in its identity representation on $H_{1}$ with $H_{1}$ (respectively $H_{2}$ ) and $\ell^{2}\left(\mathbb{F}_{2}\right)$ identified so that $\left\{\xi_{2 i}: i \in \mathbb{N}\right\}$ (respectively $\left.\left\{\xi_{2 i-1}: i \in \mathbb{N}\right\}\right)$ is the standard basis of $\ell^{2}\left(\mathbb{F}_{2}\right)$ in some enumeration, and let $A_{0}$ be the non-unital $\mathrm{C}^{*}$-algebra $u B u+K(H)$. Then $A_{0} / K\left(\ell^{2}\left(\mathbb{F}_{2}\right)\right) \cong C_{r}^{*}\left(\mathbb{F}_{2}\right)$.

For $t=\lambda_{g} \in C_{r}^{*}\left(\mathbb{F}_{2}\right)$ and $i \in \mathbb{N}$,

$$
t_{i i}=\left(t \xi_{2 i} \mid \xi_{2 i}\right)=\left\{\begin{array}{ll}
1 & (g=e) \\
0 & (g \neq e)
\end{array},\right.
$$

where $e$ denotes the identity element of $\mathbb{F}_{2}$. Moreover

$$
e_{2 i} u(t \oplus 0) u e_{2 i}=\frac{1}{2} t_{i i} e_{2 i, 2 i}
$$

and

$$
e_{2 i-1} u(t \oplus 0) u e_{2 i-1}=\frac{1}{2} t_{i i} e_{2 i-1,2 i-1}
$$

for $t \in C_{r}^{*}\left(\mathbb{F}_{2}\right)$ and $i \in \mathbb{N}$. Let $C_{0}$ be the abelian $\mathrm{C}^{*}$-algebra generated by the set of projections $\left\{e_{i i}: i \in \mathbb{N}\right\}$ in $A_{0}$. The weak-operator closure $L$ of $C_{0}$ is maximal abelian in $B(H)$ and if $\sigma$ is the canonical projection onto $L$, then for $t \in C_{r}^{*}\left(\mathbb{F}_{2}\right)$,

$$
\sigma(u(t \oplus 0) u)=\sum_{i} e_{i} u(t \oplus 0) u e_{i}=\frac{1}{2} \sum_{i} t_{i i}\left(e_{2 i, 2 i}+e_{2 i-1,2 i-1}\right)=\lambda 1,
$$

for some $\lambda \in \mathbb{C}$, where 1 is the identity operator on $H$. If $k+b \in C_{0}^{c}$ with $k \in K(H)$ and $b \in u B u$, then $k+b \in L^{c}=L$ and so $k+b=\sigma(k)+\sigma(b)$. Now $\sigma(k) \in C_{0}$ and $\sigma(b)=\lambda 1$ for some $\lambda \in \mathbb{C}$. Hence $\lambda 1 \in A_{0}$. Since $A_{0}$ is non-unital, $\lambda=0$, which implies that $k+b=\sigma(k) \in C_{0}$. Thus $C_{0}^{c}=C_{0}$ and $C_{0}$ is maximal abelian in $A_{0}$.

Let $q: A_{0} \rightarrow C_{r}^{*}\left(\mathbb{F}_{2}\right)$ be the quotient map. With $\lambda$ and $\rho$ as in 3.1, $\{\lambda, \rho \circ q\}$ is a commuting pair of representations of the pair $\left\{C_{r}^{*}\left(\mathbb{F}_{2}\right), A_{0}\right\}$ on $\ell^{2}\left(\mathbb{F}_{2}\right)$ with corresponding representation $\pi_{r}$ of $C_{r}^{*}\left(\mathbb{F}_{2}\right) \odot A_{0}$ given by

$$
\pi_{r}\left(\sum a_{i} \otimes b_{i}\right)=\sum \lambda\left(a_{i}\right) \rho\left(q\left(b_{i}\right)\right)
$$


A C*-norm \|\|$_{\alpha}$ on $C_{r}^{*}\left(\mathbb{F}_{2}\right) \odot A_{0}$ is defined by

$$
\|x\|_{\alpha}=\max \left\{\|x\|_{\min },\left\|\pi_{r}(x)\right\|\right\} \quad\left(x \in C_{r}^{*}\left(\mathbb{F}_{2}\right) \odot A_{0}\right) .
$$

As in the proof of Proposition 4, for any norm \|\|$_{\beta}$ on $C_{r}^{*}\left(\mathbb{F}_{2}\right) \odot A_{0}$ with $\|x\|_{\beta} \geq$ $\|x\|_{\alpha}$, there is a non-zero $x \in C_{r}^{*}\left(\mathbb{F}_{2}\right) \otimes_{\beta} A_{0}$ such that $\Psi(x)=0$, where $\Psi$ is the canonical homomorphism from $C_{r}^{*}\left(\mathbb{F}_{2}\right) \otimes_{\beta} A_{0}$ to $C_{r}^{*}\left(\mathbb{F}_{2}\right) \otimes_{\min } A_{0}$. Since $K(H)$ is nuclear, $\left.\Psi\right|_{C_{r}^{*}\left(\mathbb{F}_{2}\right) \otimes_{\beta} K(H)}$ is isometric and, as in the proof of Proposition 4, [x,a®k]=0 for any $a \in C_{r}^{*}\left(\mathbb{F}_{2}\right)$ and $k \in K(H)$. Thus $x \in\left(C_{1} \otimes C_{0}\right)^{c} \backslash\left(C_{1} \otimes C_{0}\right)$ for any masa $C_{1}$ in $C_{r}^{*}\left(\mathbb{F}_{2}\right)$, in particular if $C_{1}$ is the abelian $C^{*}$-subalgebra of $C_{r}^{*}\left(\mathbb{F}_{2}\right)$ with the extension property generated by one of the canonical unitary generators. It is easy to see directly that $C_{0}$ does not contain an approximate identity for $A_{0}$.

4. Some open problems. When $H$ is the Hilbert space $\ell^{2}(\mathbb{N}), B(H)$ is non-nuclear and in particular the $\mathrm{C}^{*}$-norms \|\|$_{\max }$ and \|\|$_{\min }$ on $B(H) \odot C_{r}^{*}\left(\mathbb{F}_{2}\right)$ are distinct [13]. If $C$ is a non-atomic masa of $B(H)$ isomorphic to $L^{\infty}(0,1)$ (which does not have the extension property by [9]), is it true that $(C \otimes 1)^{c}=C \otimes C_{r}^{*}\left(\mathbb{F}_{2}\right)$ in $B(H) \otimes_{\max } C_{r}^{*}\left(\mathbb{F}_{2}\right)$ ? Junge and Pisier [7] have shown that the $C^{*}$-norms \|\|$_{\max }$ and \|\|$_{\min }$ on $B(H) \odot B(H)$ are distinct. Is it true that $(C \otimes 1)^{c}=C \otimes B(H)$ in $B(H) \otimes_{\max } B(H)$ for any masa $C$ of $B(H)$ ? Is $C_{1} \otimes C_{2}$ maximal abelian in $B(H) \otimes_{\max } B(H)$ for any masas $C_{1}$ and $C_{2}$ ? The case $C_{1} \cong C_{2} \cong \ell^{\infty}(\mathbb{N})$ is particularly intriguing. The question of whether masas isomorphic to $\ell^{\infty}(\mathbb{N})$ have the extension property relative to $B(H)$ was first investigated by Kadison and Singer [9], but remains at the time of writing one of the more significant unsolved problems in the subject, despite the attention of many distinguished workers. If the Kadison-Singer problem had a positive solution, it would follow by Corollary 3 that $\ell^{\infty}(\mathbb{N}) \otimes \ell^{\infty}(\mathbb{N})$ is maximal abelian in $B(H) \otimes_{\max } B(H)$. If however it could be shown that $\ell^{\infty}(\mathbb{N}) \otimes \ell^{\infty}(\mathbb{N})$ is not maximal abelian in $B(H) \otimes_{\max } B(H)$, then it would follow that $\ell^{\infty}(\mathbb{N})$ does not have the extension property.

Acknowledgement. The author is grateful to Stuart White for a stimulating discussion on the topics considered here and to the referee for pointing out a number of inaccuracies.

\section{REFERENCES}

1. C. A. Akemann and F. Shultz, Perfect $C^{*}$-algebras, Mem. Amer. Math. Soc. 55 (1985), no. 326

2. J. Anderson, A maximal abelian subalgebra of the Calkin algebra with the extension property, Math. Scand. 42 (1978), 101-110.

3. R. J. Archbold, On the centre of a tensor product of $C^{*}$-algebras, J. London Math. Soc. (2) 10 (1975), 257-262. $351-354$.

4. R. J. Archbold, Extensions of states of $C^{*}$-algebras, J. London Math. Soc. (2) 21 (1980),

5. C. J. K. Batty, On relative commutants in tensor products of $C^{*}$-algebras, Math. Z. 151 (1976), 215-218.

6. R. G. Haydon and S. Wassermann, A commutation result for tensor products of $C^{*}$ algebras, Bull. London Math. Soc. 5 (1973), 283-287.

7. M. Junge and G. Pisier, Bilinear forms on exact operator spaces and $B(H) \otimes B(H)$, Geom. Funct. Anal. 5 (1995), 329-363. 
8. E. C. Lance, Tensor products of non-unital $C^{*}$-algebras, J. London Math. Soc. (2) 12 (1975/76), 160-168.

9. R. V. Kadison and I. M. Singer, Extensions of pure states, Amer. J. Math. 81 (1959), $383-400$

10. S. Sakai, $C^{*}$-algebras and $W^{*}$-algebras, Ergebnisse der Mathematik und ihrer Grenzgebiete, Band 60 (Springer-Verlag, 1971).

11. M. Takesaki, On the cross-norm of the direct product of $C^{*}$-algebras, Tôhoku Math. J. (2) 16 (1964), 111-122.

12. S. Wassermann, The slice map problem for $C^{*}$-algebras, Proc. London Math. Soc. (3) 32 (1976), 537-559.

13. S. Wassermann, On tensor products of certain group $C^{*}$-algebras, J. Functional Analysis 23 (1976), 239-254.

14. S. Wassermann, Tensor products of free-group C*-algebras, Bull. London Math. Soc. 22 (1990), 375-380. 